\title{
RISE-Based Adaptive Control for EICoSI Exoskeleton to Assist Knee Joint Mobility
}

\author{
Kashif I K Sherwani ${ }^{\#}$ \\ Jamia Millia Islamia, \\ Department of Electrical Engineering, New Delhi, India \\ kashifsherwani18@gmail.com \\ Neelesh Kumar \\ Central Scientific Instruments Organisation, \\ Chandigarh, India \\ neel5278@gmail.com
}

\author{
Ahmed Chemori \\ LIRMM - UMR 5506 - CC 477 \\ 161 rue Ada \\ 34095 Montpellier Cedex 5, France \\ Ahmed.Chemori@lirmm.fr
}

\section{Munna Khan}

Jamia Millia Islamia,

Department of Electrical Engineering, New Delhi, India

mkhan4@jmi.ac.in

\section{Samer Mohammed}

Laboratory of Images, Signals and Intelligent Systems (LISSI)

University of Paris-Est, Créteil (UPEC), IUT Créteil-Vitry, France

samer.mohammed@u-pec.fr

\# Corresponding Author 


\title{
Highlights
}

- An adaptive RISE control strategy is developed for the flexion/extension of the knee joint

- Stability analysis of the human-exoskeleton system is provided using Lyapunov theory

- Comparative analysis is performed between standard adaptive, RISE and adaptive RISE controllers

- Different environment conditions are applied to the human-exoskeleton system to check the robustness of the controller

\begin{abstract}
Exoskeleton devices are used to assist joint motion of subjects suffering from mobility deficiencies. Controlling an exoskeleton subjects to high nonlinearities, which are mainly due to the mechanical coupling, external disturbances, parameter uncertainties, and modeling errors. Keeping in view the requirement of a relatively accurate movement tracking while reducing the disturbances effects, there is a need for a robust controller. In this paper, an adaptive RISE (Robust Integral of Sign Error) controller is developed and implemented on the EICoSI (Exoskeleton Intelligently Communicating and Sensitive to Intention) knee exoskeleton. RISE has an advantage over standard controllers that it achieves semi-global asymptotic tracking even in the presence of unstructured disturbances. But to achieve this tracking, high control gains are required. Thus, to limit such high gains, RISE control strategy is combined with an adaptive controller, which has the advantage of improving the tracking performance while reducing the eventual overshoots. The stability of the coupled human/ exoskeleton system is analyzed based on Lyapunov theory and the system has shown semi-global asymptotic stability. The adaptive RISE controller gives better SNR (signal to noise ratio) by $11 \%$ and $2 \%$ as compared with adaptive and RISE controller respectively. In terms of tracking error, the adaptive RISE controller shows 9\% more error than adaptive controller but $41 \%$ less when compared with RISE controller. Three experimental scenarios are analyzed to validate the proposed controller i.e. (i) external disturbances, that could come from the ground during walking; (ii) induced payload, that could come from the resistive/assistive torque from the muscles and (iii) payload with external disturbances. The system is found to be robust and efficient in tracking the reference trajectories while maintaining limited error and high signal to noise ratio.
\end{abstract}

Keywords: Adaptive Controller, Asymptotic Stability, RISE Feedback, Knee Exoskeleton

\section{Introduction}

Joint mobility limitations are the common effects resulting from spinal cord injuries and strokes. Conventional intensive therapy by at least two rehabilitation operators, is often used in clinics to recover the voluntary movements. Such methods are effective when it is intense and also requires an active participation of patients but at the same time, it is very tedious and time consuming 
for the clinical staffs while repeatability of the treatment is not guaranteed. Exoskeletons are mechanical devices driven by the actuator to transfer the generated torque to the patient's joints and provides an effective solution for movement restoration to the patients enabling long repetitive rehabilitation sessions. Such devices were initially developed for military purposes to carry heavy loads such as the Berkeley Lower Extremity Exoskeleton (BLEEX) [1,2] that allows the wearer to carry heavy loads. Initial work in exoskeletons for the lower limb rehabilitation was carried out by Vukobratovic et. al. [3]. Later, HAL [4] was developed for the rehabilitation of lower limbs with controlled hip and knee joints and passive ankle joint. Recently, Walkbot [5][6] was developed to assist the lower limb joints (i.e. hip, knee, and ankle). To provide interactive training and monitoring, it uses $3 \mathrm{~d}$ virtual reality and pressure plates. ATALANTE [7] is another example of lower limb exoskeleton with 12 actuated joints. It supports crutch-less dynamic walking. Whole body exoskeletons are complex and there is a need of single joint exoskeleton. Keeogo [8] is the knee joint exoskeleton to assist walk, run, climb, kneel and squat. Leg [9] is another knee exoskeleton to provide patients with repeated cycle of therapy.

The knowledge of the joint dynamics in the control structure is important for effective control. During gait or sit to stand, knee joint contributes upto 70 percent of the mechanical work. The strength i.e. torque generated by the muscles actuating the knee joint determines the effectiveness of the patient's daily living activities. This paper proposes an adaptive RISE (Robust Integral of Sign Error) control approach to assist the knee joint exoskeleton achieving the flexion/extension movements while guaranteeing minimal tracking error with respect to the desired trajectory. Classical proportional-integral (PI), proportional derivative (PD) or proportional integral derivative (PID) controllers are widely used in literature either alone or with the combination of other controllers. HAL 5 [10] is used to assist elderly and disabled through a bipedal locomotion system (provides assistance to both legs). PD control is applied for controlling the walking trajectories or motion support phase synchronization with patient's intention calculated from EMG signals. For the tracking control in swing phase, the gait pattern of the healthy person is used as a reference in control architecture, and in landing and support phase, it uses the constant control value. RoboKnee [11] also uses PD controller to control the linear actuator and was designed to assist human locomotion with one degree of freedom. It evaluates vertical ground reaction forces to calculate the user's intention and assist thigh muscles while performing flexion-extension of the knee joint. The controller here amplifies the human torque when the desired trajectory is not defined. TUPLEE [12] also amplifies the knee joint torque, by taking EMG signals as an input from the thigh muscles. The problem or the limitation with the amplification technique is that it depends on the muscle fatigue for the estimation of the force. Active knee rehabilitation orthotic device (AKROD) [13] uses PI controller to actuate the Electro-Rheological Fluid (ERF) based actuator and provides resistive torque. But it didn't give the satisfactory initial transient response due to ERF material property and unmodeled dynamic system behavior. So, an adaptive, as well as step logic function, is added to the PI controller to overcome this problem. Costa et. al. [14] proposed a PID based controller 
to control the actuation of a high-power pneumatic muscle actuator for a lower limb orthosis having five DoF on each leg. It faces the problem of occasional nonlinearities. Cyberthosis [15] is a hybrid knee exoskeleton with PID as a feedforward controller for the knee joint flexion/extension by applying functional stimulator. Saito et. al. [16] also proposed a PID controller for bilateral servo actuator having a biarticular muscle mechanism to overcome the problem of noise, speed and weight in the externally powered orthosis. The above said systems did not consider the physical constraints of the exoskeleton that helps in avoiding the actuator's saturation causing the instability in the exoskeleton system. The classical linear controllers have the drawback that it did not consider the system nonlinearities and are not sufficiently robust with respect to external disturbances. The exoskeleton along with the patient's muscle dynamics forms a very complex scenario and it makes the classical controllers inefficient and inaccurate in the presence of uncertainties and external disturbances.

Many other control strategies have also been proposed in the literature. Hayashi et. al. [17] proposed an adaptation technique based on neuro-fuzzy controller for a three-DoF lower limb exoskeleton using EMG signals as input signals. Kigushi et. al. [18] also used the EMG as input signals for the hierarchical neuro-fuzzy based approach to control an upper limb exoskeleton which has shown more efficiency than the conventional neuro-fuzzy controller but did not prove the stability of the system. Admittance/Impedance-based control [19][20] and counterbalance [21][22] are also used as assistance control strategies.

Fuzzy or neural network [23][24] approaches are used to approximate the uncertain dynamics but still, external disturbances do affect the tracking results. Some researchers used sliding mode controllers (SMC) [25][26] to control the exoskeleton. Two types of controllers are proposed by Banala et al. [27]. A basic SMC, for orthosis movement in a prescribed pattern and a feedback linearizing controller, to provide the additional torque required to move the orthosis to the desired trajectory. Weinberg et al. [28] proposed the use of the two controllers for active knee rehabilitation, the first one is the hybrid PI controller for robust closed-loop control torque and the second one uses the SMC to control the closed-loop velocity. The basic sliding mode technique achieves asymptotic tracking for uncertain nonlinear systems, but it yields discontinuous control due to the chattering effect, which may be harmful to the actuators. To overcome this problem Mohammed et. al. [29] introduces the nonlinear observer to the controller and was used for the estimation of muscle torque.

Adaptive controllers [30][31] can also be used for the asymptotic stable result. To compensate for dynamic uncertainties, Peternel et. al. [32] uses adaptive learning methodology to control the arm joint torque. It has been observed that the system with higher unstructured uncertainty requires controllers with more control effort. Higher control effort includes high-frequency feedback or higher gain, that results in reduced performance of the system. Chen et. al. [33] proposed an adaptive cascade force control strategy and grouped it into two control levels i.e. high and low to drive a one DoF hydraulic exoskeleton, the high level estimates the human motion intention while the low-level one contains the motion tracking algorithm. 
The Robust Integral of the Sign of the Error (RISE) [34] is a high gain feedback control strategy that has a unique integral signum term that can adjust smooth bounded disturbances. The advantage of this control scheme is that the asymptotic stability can be achieved even in the presence of uncertainties and disturbances. Cai et. al. [35] developed a tracking controller for nonlinear systems with parametric uncertainties and disturbances. It has the problem of over parameterization that increases the dynamic order of the controller and affects the system robustness. The first robust controller [36] was proposed by Xian et. al. [37] and implemented on a class of nonlinear system having continuous differentiable dynamics. Several control methodologies have been designed using RISE controllers for underwater vehicle [38] and flight control applications [39]. The major drawback of the RISE controllers is that it requires a high gain condition and reduced performance is also observed in the presence of unstructured uncertainties. To improve the performance and reduce the effects of the high gain, RISE feedback is generally fused with other controllers such as Neural Network [40][41]. Adding such controllers ensures the knowledge of the system dynamics and helps to reduce the tracking error. Xian et. al. [42] concluded that the use of RISE controller in conjunction with a feedforward component (constant best guess) can yield a uniformly ultimately bounded result. For systems satisfying the linear-in-the-parameters assumptions, Patre et. al. [40] combines the standard gradient feedforward term with the RISE controller and observes the improvement in the RMS (root mean square) tracking error with reduced control effort.

The adaptive controller has the advantage that it can compensate dynamic uncertainties, but it has a drawback that it needs higher control gain. On the other hand, the RISE controller can compensate smooth bounded disturbances but in the presence of unstructured disturbances, it shows reduced performance. So, in this paper, a RISE controller is combined with an adaptive controller and applied to the knee joint exoskeleton to ensure flexion/extension movements, to compensate the drawbacks and exploit the advantages of both the controllers as explained in section III. The stability of the system has been proved based on the Lyapunov theory which guarantees safety to the wearer. The controller is then experimentally validated in different experimental conditions (i.e. with varying payload and externally induced disturbances) to show the robustness of the proposed controller. Comparative analysis is also presented between the adaptive, RISE and adaptive RISE controller in terms of tracking error and SNR (signal to noise ratio). The paper is organized as follows: Section II explains the modeling of the knee exoskeleton system. In this section, the dynamics of the human-leg exoskeleton are derived. Section III is devoted to the development of the proposed adaptive RISE control for knee exoskeleton. Here, the adaptive and RISE controllers are also presented along with its limitations. Section IV describes the experimental setup (i.e. knee exoskeleton) used for the experiment and identification of the exoskeleton shank parameters. The control parameters are also presented in this section along with its sensibility performance. Section V shows the experimental results of the implementation of the proposed adaptive RISE controller with varying payload and external disturbances. To check the robustness of the controller five different experimental conditions are taken and presented in this section. 
Comparative analysis of the different controllers in terms of tracking error and SNR (signal to noise ratio) are also done. Finally, Section VI concludes the paper. Stability analysis of the adaptive RISE controller is also proved using Lyapunov stability approach and presented in annexure at the end of the paper.

\section{Shank Knee Exoskeleton Modeling}

In this study, the experiments were conducted using the EICoSI (Exoskeleton Intelligently Communicating and Sensitive to Intention) [43], developed at the LISSI Laboratory (Laboratoire Images, Signaux et Systèmes Intelligents) of University Paris-Est Créteil (UPEC), France. The knee joint acts as a pendulum having two segments moving along the rotational axis as shown in Fig. 1. The upper part behaves as a thigh, fixed along the $\mathrm{x}$-axis and driven by the actuator allowing flexion/extension of the knee joint whereas the lower end behaves as shank-foot attached with the exoskeleton with its movement constrained between $0^{\circ}$ and $120^{\circ}$. Both human leg and exoskeleton are supposed to have the same rotational degree of freedom around the knee joint. The torque generated by the actuator computed by means of the controller ensures the desired flexion/extension of the knee joint.

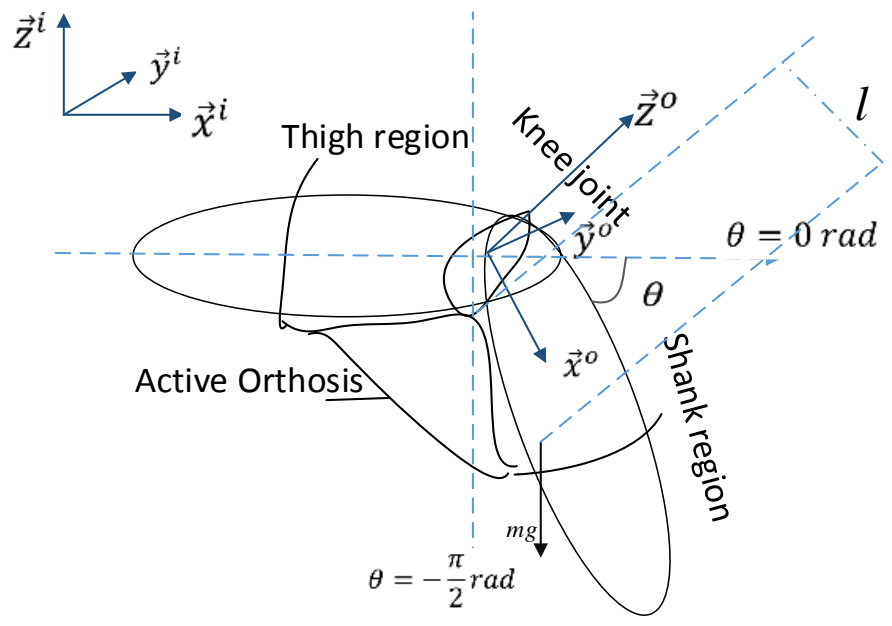

Fig. 1 Lower Limb Exoskeleton Model

Two frames are defined in which the knee joint moves, global frame $\left(\vec{x}^{i}, \vec{y}^{i}, \vec{z}^{i}\right)$ which is earth-centered fixed frame and the exoskeleton frame $\left(\vec{x}^{o}, \vec{y}^{o}, \vec{z}^{o}\right)$ that coincides and moves with the knee joint at an angle $\theta$ such that $\vec{y}^{i}$ and $\vec{y}^{o}$ coincides during orientation. Since the knee joint has one degree of freedom, angular velocity $\dot{\theta}$ denotes derivative of $\theta$ of the knee joint.

The dynamic model of both human leg and exoskeleton are derived simultaneously [43]. The Lagrangian of the coupled system denotes the dynamics of both the human leg and exoskeleton

$$
\begin{gathered}
\mathcal{L}_{i}=E_{k_{i}}-E_{g_{i}} \\
\mathcal{L}_{i}=E_{k_{i}}-E_{g_{i}}
\end{gathered}
$$

$E_{k_{i}}$ and $E_{g_{i}}$ denotes the kinetic and gravitational energies of the system component respectively and $i \epsilon(1,2)$ for the human leg 
and exoskeleton.

$$
E_{k_{i}}=\frac{1}{2} J_{i} \dot{\theta}^{2}
$$

where $J_{i}$ is the inertia of the human leg and exoskeleton

$$
E_{g_{i}}=m_{i} g l_{i}(1-\sin \theta)
$$

$m_{i}$ is the mass of the human leg and exoskeleton

$g$ is the acceleration due to gravity

$l_{i}$ is the distance between the knee joint and center of gravity

on deriving $\mathcal{L}_{i}$, the resulting dynamics of the system can be written as

$J_{i} \ddot{\theta}=m_{i} g l_{i} \cos \theta-\tau_{\text {ext }_{i}}$

where $\tau_{\text {ext }}$ shows the external torque applied to the system.

$$
\tau_{\text {ext }}=\tau_{f_{i}}+\tau_{i}
$$

$\tau_{f_{i}}$ and $\tau_{i}$ are the friction and motor control torque respectively, with

$$
\tau_{f_{i}}=-A_{i} \operatorname{sign} \dot{\theta}-B_{i} \dot{\theta}
$$

$A_{i}$ and $B_{i}$ are the solid and viscous friction coefficients respectively.

The system model consisting of the human leg and exoskeleton is given by

$$
J \ddot{\theta}=-\tau_{g} \cos \theta-A \operatorname{sign} \dot{\theta}-B \dot{\theta}+\tau_{c}+\tau_{h}
$$

where, $J=\sum_{i=1}^{2} J_{i}, \tau_{g}=\sum_{i=1}^{2} \tau_{g_{i}}, A=\sum_{i=1}^{2} A_{i}$ and $B=\sum_{i=1}^{2} B_{i}$.

$\theta, \dot{\theta}, \ddot{\theta}$ represent the angular position, velocity, and acceleration of the coupled system respectively.

$J, A, B, \tau_{g}, \tau_{c}, \tau_{h}$ are Inertia, solid friction coefficient, viscous friction coefficient, gravity torque, controller torque and human torque of the human leg and exoskeleton respectively. The human leg-exoskeleton parameters are identified in section 4.1.

The equation (2.7) can be represented in standard joint space form as

$$
M(\theta) \ddot{\theta}+f(\theta, \dot{\theta})+G(\theta)=B(u)
$$

where, $M(\theta)$ = inertia matrix,

$$
\begin{aligned}
& f(\theta, \dot{\theta})=\text { friction force, } \\
& G(\theta)=\text { gravity force } \\
& B(u)=\text { torque acting at the knee joint. }
\end{aligned}
$$

\section{Exoskeleton Control}


The EICoSI exoskeleton is a nonlinear system and is subjected to various uncertainties like nonlinear coupling, parameter perturbations and also suffers from external disturbances. Therefore, effective control is an essential element in exoskeleton design to ensure the accuracy of the non-linear system. Controllers like proportional or PID provides an attractive control solution for real-time implementation because of its simplicity [44]. To diminish the effects of disturbances, proportional controller requires high control gain, whereas in PID, the integral term effectively overcomes the constant disturbances but does not perform well with time-varying disturbances [45]. Therefore, there is a need for more reliable and robust control design. Adaptive control effectively overcomes the uncertainties in model parameters and is successfully implemented in real-time control of exoskeleton devices [43][46][47][48]. It provides a systematic approach through control laws and is quite helpful in unknown or with timevarying process dynamics.

In this study, the objective of the controller is to ensure accurate tracking of the knee joint desired trajectory while guaranteeing limited or no overshoot in the case of eventual external disturbances. Such disturbances occur for example due to the voluntary torque generated by the wearer's muscles or resulting from the impact with the ground while walking.

\subsection{Nonlinear Adaptive Control}

Nonlinear Adaptive Control are widely used for controlling exoskeleton devices [49][50][51] due to its robustness with respect to the environmental and model uncertainties. This control strategy ensures accurate tracking errors even in the presence of change in the dynamics of the exoskeleton. The adaptive parameters (example: friction, inertia, and gravity) are continuously updated in order to reduce the error as much as possible between the desired and the current trajectory.

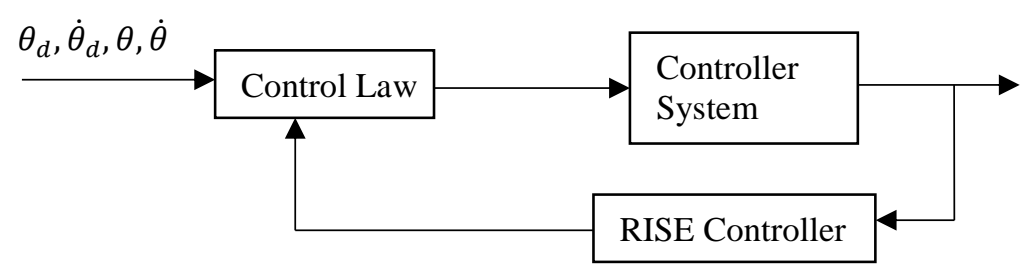

Fig. 2 Block diagram of adaptive controller

The adaptive controller [43] as shown in Fig. 2 is defined as

$$
\begin{aligned}
& \tau_{a}=\hat{J}\left(\ddot{\theta}_{d}-\lambda \dot{\tilde{\theta}}\right)+\hat{A} \operatorname{sign} \dot{\theta}+\hat{B} \dot{\theta}-k s+\hat{\tau}_{g} \cos \theta \\
& \dot{\hat{J}}=-a_{1}\left(\ddot{\theta}_{d}-\lambda \dot{\tilde{\theta}}\right) s ; \dot{\hat{A}}=-a_{2} \operatorname{sign} \dot{\theta} s ; \dot{\hat{B}}=-a_{3} \dot{\theta} s ; \dot{\hat{\tau}}_{g}=-a_{4} \cos \theta s
\end{aligned}
$$

$\dot{\hat{J}} ; \dot{\hat{A}} ; \dot{\hat{B}} ; \dot{\hat{\tau}}_{g}$ are the adaptive parameters and the hat represent its estimate.

$$
\tilde{\theta}=\theta-\theta_{d} ; \dot{\tilde{\theta}}=\dot{\theta}-\dot{\theta}_{d} ; \ddot{\tilde{\theta}}=\ddot{\theta}-\ddot{\theta}_{d}
$$

$(\tilde{\theta}, \dot{\tilde{\theta}}, \ddot{\tilde{\theta}}$ are the error between current and desired angle, angular velocity, and acceleration respectively) 


$$
s=\dot{\tilde{\theta}}+\lambda \tilde{\theta} ; \ddot{\theta}=\dot{s}-\lambda \dot{\tilde{\theta}}+\ddot{\theta}_{d}
$$

$\mathrm{s}$ is variable with $\lambda$ as positive scalar

Using equation (2.8), the system dynamics could be expressed as follows:

$$
M(\theta) \ddot{\theta}+f(\theta, \dot{\theta})+G(\theta)=Y(\theta, \dot{\theta}, \ddot{\theta}) \emptyset
$$

where $\mathrm{Y}(\cdot) \in R^{n \times p}$ is a nonlinear function known as regression matrix and is a function of position, velocity and acceleration of the knee joint and $\emptyset=\left[\emptyset_{1}, \emptyset_{2}, \emptyset_{3}, \ldots, \emptyset_{p}\right] \in R^{p}$ is the knee joint vector parameter to be estimated.

\subsection{RISE Controller}

The problem with adaptive control is that it requires high control torque that needs to be avoided for the wearer's safety as the actuator's saturation results in undesirable behavior. Also, compensating the friction to reformulate the control law is not recommended as it results in a relative high load of the actuator causing an unpredictable behavior of an exoskeleton.

Robust Integral of the Sign of the Error (RISE) is a control strategy with differentiable high gain feedback and contains integral signum function in the dynamics of the controller for smooth bounded disturbances. The advantage of this controller is that it can achieve asymptotic stability despite the presence of uncertainties and disturbances. The controller can be used as a tracking controller for systems having nonlinear behavior with time derivative bounded parametric uncertainties and disturbances [52].

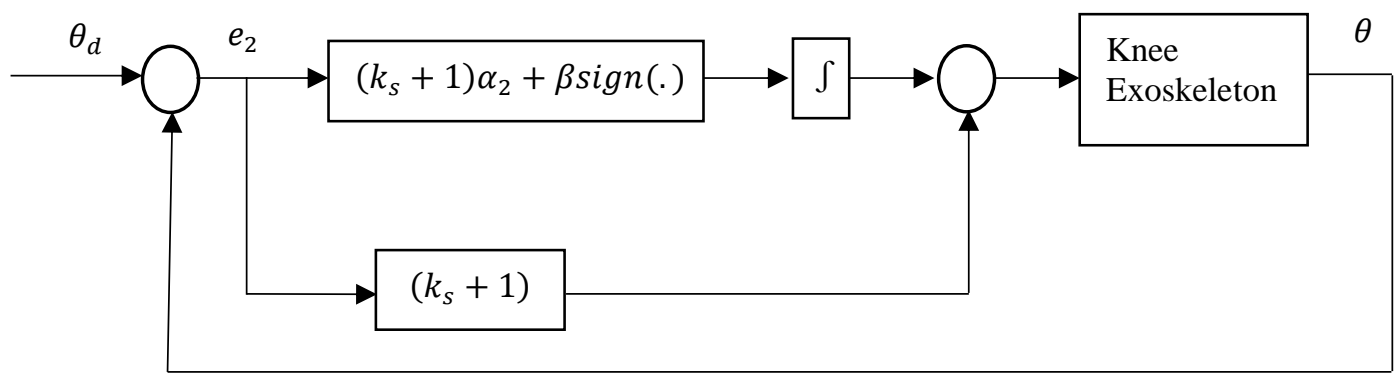

Fig. 3 Block diagram of RISE controller

The block diagram of the RISE controller is shown in Fig. 3. To quantify the error i.e. control objective, the tracking error $e_{1}$ $\left(e_{1} \in R^{n}\right)$ is defined as

$$
e_{1}=\theta_{d}-\theta=-\tilde{\theta}
$$

And the auxiliary error is defined as $e_{2} ;\left(e_{2} \in R^{n}\right)$, to facilitate the control design

$$
\begin{aligned}
& e_{2}=\dot{e}_{1}+\alpha_{1} e_{1}=-\left(\dot{\tilde{\theta}}+\alpha_{1} \tilde{\theta}\right) \\
& \mathrm{r}=\dot{e}_{2}+\alpha_{2} e_{2}
\end{aligned}
$$

where, $\alpha_{1}$ and $\alpha_{2}$ are positive control design parameters.

The controller is defined considering the initial conditions to be zero as 


$$
\tau_{r}=\left(k_{s}+1\right) e_{2}-\left(k_{s}+1\right) e_{2}(0)+v_{f}
$$

where $v_{f}\left(v_{f} \in R^{n}\right)$ is the Filippov solution of the differential equation

$$
\dot{v}_{f}=\left(k_{s}+1\right) \alpha_{2} e_{2}+\beta \operatorname{sign}\left(e_{2}\right)
$$

$k_{s}, \beta \in \mathrm{R}$ are constant positive control gains.

The open-loop tracking error is derived by multiplying $\mathrm{M}(\theta)$, the inertia matrix, to equation (3.8) and using equation (2.8).

$$
M(\theta) r=G(\theta)+f(\theta, \dot{\theta})+M(\theta)\left(\ddot{\theta}_{d}+\alpha_{1} \dot{e}_{1}+\alpha_{2} e_{2}\right)-B(u)
$$

On taking first-time derivative of equation (3.11) and using equation (3.9) and (3.10).

$$
M(\theta) \dot{r}=-\frac{1}{2} \dot{M}(\theta) r+N(\cdot)-\dot{\tau}_{r}-e_{2}
$$

where, $N(\cdot)$ is an unmeasurable nonlinear auxiliary function.

$$
\begin{aligned}
& N(\cdot)=\dot{M}(\theta)\left(\alpha_{1}\left(e_{2}-\alpha_{1} e_{1}\right)+\alpha_{2} e_{2}\right)+M(\theta)\left(\alpha_{1}\left(r-\alpha_{2} e_{2}-\alpha_{1}\left(e_{2}-\alpha_{1} e_{1}\right)\right)+\alpha_{2}\left(r-\alpha_{2} e_{2}\right)\right)+\dot{M}(\theta) \ddot{\theta}_{d}+M(\theta) \theta_{d}^{(3)}+ \\
& \dot{G}(\theta, \dot{\theta})+\dot{f}(\theta, \dot{\theta})+e_{2}-\frac{1}{2} \dot{M}(\theta) r
\end{aligned}
$$

Consider another auxiliary function,

$$
N_{d}(\cdot)=\dot{M}\left(\theta_{d}\right) \ddot{\theta}_{d}+M\left(\theta_{d}\right) \theta_{d}^{(3)}+\dot{G}\left(\theta_{d}\right)+\dot{f}\left(\theta_{d}, \dot{\theta}_{d}\right)
$$

Using equations (3.12), (3.13) and (3.14),

$$
M(\theta) \dot{r}=-\frac{1}{2} \dot{M}(\theta) r+\dot{\tau}_{r}-e_{2}+\widetilde{N}(\cdot)+N_{d}(\cdot)
$$

$\widetilde{N}(\cdot)=N(\cdot)-N_{d}(\cdot)$ can be upper bounded using the final value theorem

$$
\|\widetilde{N}(t)\| \leq \rho(\|z\|)\|z\|
$$

where $\mathrm{z}(\mathrm{t}) \in \mathrm{R}^{(\mathrm{n}+1) \mathrm{m}}$ is defined as

$$
z=\left[\begin{array}{lllll}
e_{1}^{T} & e_{2}^{T} & \ldots & e_{n}^{T} & r^{T}
\end{array}\right]^{T}
$$

and $\rho(\|z\|) \in R$ is the globally invertible, positive, non-decreasing known bounding function.

\subsection{Adaptive RISE Controller}

In a RISE based controller, the system uncertainties can be compensated by using high feedback gains. The systems that have dynamics and can be differentiated between unstructured and structured uncertainties, the RISE based system is used in conjunction with the adaptive to achieve the asymptotic tracking result. The adaptive parameters compensate the linearly parameterized uncertainties (structured) and allow the knowledge of the dynamics in the control design [40][53]. The aim is to reduce the effect of uncertainties to improve the tracking performance and helps in reducing the control effort. 
The control structure is a closed-loop system with angle and angular velocity acting as the controller feedback. The resultant torque generated from the muscle contraction because of stiffness, acts as system disturbances due to their nonlinear behavior. The task of the adaptive RISE controller is to reduce the effect of these disturbances while complementing the torque for the flexion/ extension of the knee joint and reducing the tracking error of the desired trajectory as shown in Fig. 4.

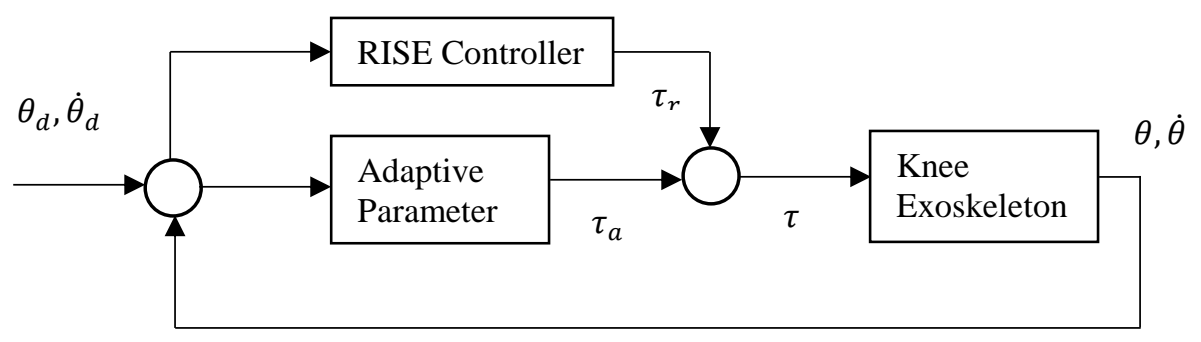

Fig. 4 Block diagram of adaptive RISE controller

The Control law of the adaptive RISE controller can be defined as

$$
\tau=\tau_{r}+Y_{d}(\cdot) \hat{\varphi}(t)
$$

where, $Y_{d}(\cdot) \epsilon R^{n \times p}$, is the desired regression matrix having desired link position functions and $\widehat{\emptyset}$ represents the parameter estimate of $\emptyset$ and follows the adaptation law as

$$
\dot{\hat{\varphi}}_{i}(t)= \begin{cases}\gamma_{i} q_{i}, \text { if } & -\varphi_{b_{i}}<\hat{\varphi}_{i}<\varphi_{b_{i}} \text { or } \\ & \hat{\varphi}_{i} \geq \varphi_{b_{i}} \text { and } q_{i} \leq 0 \text { or } \\ & \hat{\varphi}_{i} \leq-\varphi_{b_{i}} \text { and } q_{i} \geq 0 \\ 0, \quad \text { if } \quad & \hat{\varphi}_{i} \geq \varphi_{b_{i}} \text { and } q_{i} \geq 0 \text { or } \\ & \hat{\varphi}_{i} \leq-\varphi_{b_{i}} \text { and } q_{i} \leq 0\end{cases}
$$

where, $\hat{\varphi}_{i}$ is the parameter estimate of $\varphi_{i}, \gamma_{i}>0$ and $q_{i}$ is the $\mathrm{i}^{\text {th }}$ element of adaptation matrix $\Gamma \triangleq \operatorname{diag}\left(\gamma_{1}, \gamma_{2}, \ldots, \gamma_{n}\right) \in R^{n \times n}$ and column matrix $q \triangleq Y_{d}^{T}(\cdot) e_{2}$ respectively and $\varphi_{b_{i}}$ is estimated parameter bound of $\hat{\varphi}_{i}$.

The above law ensures that estimated parameters $\hat{\varphi}(t)=\left[\dot{\hat{\varphi}}_{1}(t), \dot{\hat{\varphi}}_{2}(t), \ldots, \dot{\hat{\varphi}}_{n}(t)\right] \in R^{n}$, are bounded, $-\varphi_{b} \leq\|\hat{\varphi}(t)\|_{\infty} \leq$ $\varphi_{b}, \forall t \geq 0$. To compensate for the inherent dynamics related nonlinearities, the estimated parameters will be adjusted in real-time by the adaptation law (3.19).

Using equation (3.18) and (2.8), the closed-loop error system for the adaptive RISE controller is

$$
M(\theta) r=Y_{d}(\cdot) \tilde{\varphi}(t)+W(\cdot)-\tau_{r}
$$

where $\tilde{\varphi}(t)$ is parameter estimation error and $W(\cdot)$ is an auxiliary function

$$
W(\cdot)=M(\theta)\left(\ddot{\theta}_{d}+\alpha_{1} \dot{e}_{1}+\alpha_{2} e_{2}\right)+G(\theta)+f(\theta, \dot{\theta})-Y_{d}(\cdot) \varphi
$$

On taking first derivative, and using equation (3.19), the error dynamics are

$$
e_{2}=-\frac{1}{2} \dot{M}(\theta) r+\dot{Y}_{d}(\cdot) \tilde{\varphi}+N(\cdot)-\dot{\tau}_{r}-M(\theta) \dot{r}
$$

The auxiliary function $N(\cdot)$ can be expressed as 


$$
N(\cdot)=-Y_{d}(\cdot) \dot{\hat{\varphi}}(t)+\dot{W}(\cdot)+e_{2}-\frac{1}{2} \dot{M}(\theta) r
$$

Using equation (3.22), (3.23) and adding auxiliary function $N_{d}(\cdot)$, the error dynamics will become

$$
e_{2}=-\frac{1}{2} \dot{M}(\theta) r+\dot{Y}_{d}(\cdot) \tilde{\varphi}+N(\cdot)+N_{d}(\cdot)-\dot{\tau}_{r}-M(\theta) \dot{r}
$$

The following upper bounds can be obtained from $N_{d}(\cdot)$ and $Y_{d}(\cdot)$

$$
\begin{array}{rc}
\left\|N_{d}(\cdot)\right\|_{\infty} \leq \xi_{N_{d}}, & \left\|\dot{N}_{d}(\cdot)\right\|_{\infty} \leq \xi_{N_{d_{2}}} \\
\left\|Y_{d}(\cdot)\right\|_{\infty} \leq \xi_{Y_{d}}, & \left\|\dot{Y}_{d}(\cdot)\right\|_{\infty} \leq \xi_{Y_{d_{2}}}
\end{array}
$$

The control algorithm was designed by assuming that $\theta(t), \dot{\theta}(t)$ are bounded and measurable. $M(\theta), f(\theta, \dot{\theta}), G(\theta)$ are unknown parameters.

Equation (2.8) can be represented after parameter reformulation as:

$$
M\left(\theta_{d}\right) \ddot{\theta}+f\left(\theta_{d}, \dot{\theta}_{d}\right)+G\left(\theta_{d}\right)=Y_{d}\left(\theta_{d}, \dot{\theta}_{d}, \ddot{\theta_{d}}\right) \emptyset
$$

The following assumption are considered and used for the development of the control algorithm.

Assumption 1: The inertia matrix $M(\theta)$ is positive definite, symmetric and $\forall y(t) \epsilon R^{n}$

$y^{T} M(\theta) y \epsilon\left[m_{1}\|y\|^{2}, \bar{m}(\theta)\|y\|^{2}\right]$

where $m_{1}, \bar{m}(\theta) \epsilon R$ is a known positive constant and function respectively. $\|\cdot\|$ symbolizes the standard Euclidean norm.

Assumption 2: The first and second partial derivatives of $M(\theta), f(\theta, \dot{\theta}), G(\theta)$ with respect to $\theta$ exist and bounded by known constants and $\theta(t), \dot{\theta}(t) \in \mathcal{L}_{\infty}$.

Assumption 3: The desired trajectory $\theta_{\mathrm{d}}(t)$ and its time derivatives are designed such that they belong to $\mathcal{L}_{\infty}$.

\section{Experimental Setup}

The EICoSI knee exoskeleton used in the experiments is illustrated in Fig. 5. It has two segments that can be attached to the thigh and shank region of the wearer using straps [46]. The compact brushless DC motor (BLDC motor) is used to actuate the knee joint. It was chosen owing to its light weight, reliability, robustness, operational at low frequency, reduced obstruction factor and can generate a torque of $0.129 \mathrm{Nm}$. The compact transmission system comprising ball screw, cable drive, belt transmission, and gear motor were designed to provide a relatively high torque. The pulley is actuated by a cylinder comprising ball screw and it is fixed to the exoskeleton at the knee. The rotation angle of the knee joint is determined by the incremental encoder equipped in the exoskeleton. The velocity of the knee joint is calculated numerically from its measured position. dSPACE DS1103 controller board is used to calculate the control torque. It takes the knee joint angle as an input and generates the PWM to control the velocity of an actuator. The whole actuator can provide a maximum torque of $18 \mathrm{Nm}$. Five different experimental conditions are applied on the 
exoskeleton to consider the effects of human factor and to check the robustness of the controller. The first experimental condition is when there is no payload applied on the exoskeleton, another one is when $2.5 \mathrm{Kg}$ of payload is introduced before the beginning of the flexion/extension cycle, in another condition the payload of $0.5 \mathrm{Kg}$ is introduced in between the flexion/extension cycle. The external disturbances were introduced in between the flexion/extension cycle in another condition by opposing the joint movement and in the last condition external disturbance as well as instant introduction of $0.5 \mathrm{Kg}$ payload is introduced in between the flexion/extension cycle of the exoskeleton.

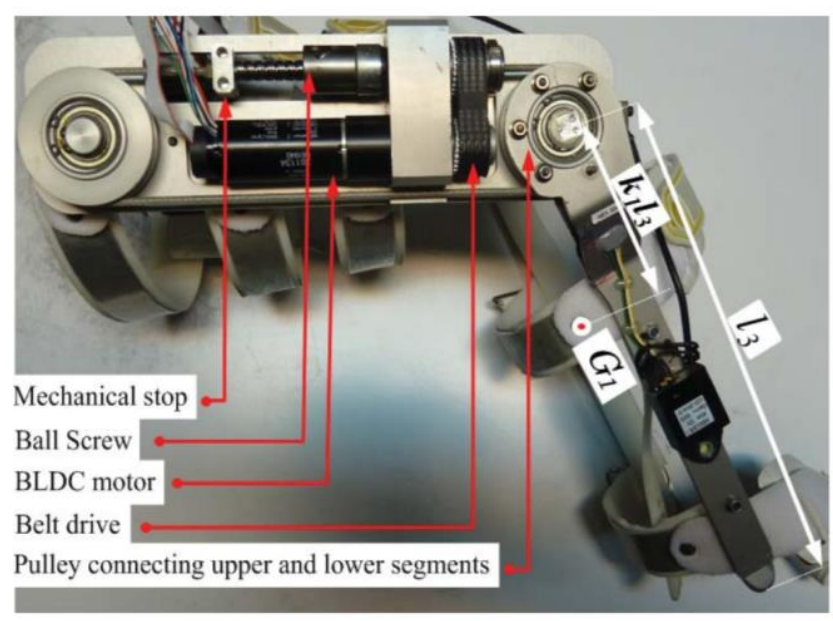

Fig. 5 View of the knee exoskeleton EICoSI and its main components [46]

\subsection{Parameters Identification}

J i.e. inertia of the system component is used to determine the kinetic energy of the system's component, A and B are the solid and viscous torque coefficient and are used to determine the friction torque and $\tau_{g}$ is the gravitational torque. Together, these parameters help in defining the system model and its dynamics. Least square method and regression equations [54][55] are used to identify the human leg-exoskeleton parameters $\left(\mathrm{J}, \mathrm{A}, \mathrm{B}, \tau_{g}\right)$. In this method, sampling of inverse dynamics model of the exoskeleton along with the excitation trajectory is done. The desired trajectory of the knee joint is used as an excitation sequence and can be represented in the form of Fourier series as

$$
q(t)=\sum_{i=1}^{n} \frac{a_{l}}{\omega_{f} l} \sin \left(\omega_{f} l t\right)-\frac{b_{l}}{\omega_{f} l} \cos \left(\omega_{f} l t\right)+q_{0}
$$

where, $\omega_{f}, \frac{a_{l}}{\omega_{f} l}, \frac{b_{l}}{\omega_{f} l}, t$ and $q_{0}$ are the fundamental radian frequency, amplitude of the sine and cosine function, time and initial value of the trajectory respectively. The Fourier series was used as it provides periodic excitation and allows averaging of the time domain. It also allows the calculation of the joint velocity and acceleration from the measured joint angle in an analytic way [56].

$$
\begin{aligned}
& -\frac{\pi}{2} \mathrm{rad} \leq \theta \leq 0 \mathrm{rad} \\
& -2.1 \mathrm{rad} / \mathrm{s} \leq \dot{\theta} \leq 2.1 \mathrm{rad} / \mathrm{s}
\end{aligned}
$$




$$
-\pi \mathrm{rad} / \mathrm{s}^{2} \leq \ddot{\theta} \leq \pi \mathrm{rad} / \mathrm{s}^{2}
$$

The above conditions reflect the real physical constraints and were used for the optimization. The position, angular velocity, acceleration and torque calculated were used in the identification of the parameter of the exoskeleton shank model [43].

The torque generated during the identification can be written as

$$
\tau_{p}=O_{p}(\theta, \dot{\theta}, \ddot{\theta}) X+r_{i}
$$

where $\tau_{p}$ is the developed torque during parameter identification, $O_{p}(\theta, \dot{\theta}, \ddot{\theta})$ is the observation matrix, $X \in R^{m}, r_{i}$ are the modeling error and measurement noise. Least square based optimization methodology is used to identify the vector of the estimated parameter $\hat{X}$.

$$
\hat{X}=\arg \min \|r\|^{2}=O_{p}^{+} \tau
$$

Where, $O_{p}^{+}=\left(O_{p}{ }^{T} O_{p}\right)^{-1} O_{p}{ }^{T} \in R^{m \times n}$.

The parameters identified for the EICoSI exoskeleton shank system are given in TABLE I. The control parameters are summarized in TABLE II and is tuned by trial and error to obtain the best results in terms of tracking error. The parameters are

\begin{tabular}{|c|c|c|c|c|}
\hline \multicolumn{5}{|c|}{$\begin{array}{c}\text { TABLE I } \\
\text { Identification Parameters } \\
\end{array}$} \\
\hline \multicolumn{4}{|c|}{ Parameter } & Value \\
\hline \multicolumn{3}{|c|}{ Inertia $(J)$} & & $0.4 \mathrm{Kg} \cdot \mathrm{m}^{2}$ \\
\hline \multicolumn{4}{|c|}{ Solid Friction Coefficient (A) } & 0.6 N.m \\
\hline \multicolumn{4}{|c|}{ Viscous Friction Coefficient (B) } & 1N.m.s.rad ${ }^{-1}$ \\
\hline \multicolumn{4}{|c|}{ Gravity Torque $\left(\tau_{g}\right)$} & $5 \mathrm{~N} . \mathrm{m}$ \\
\hline \multicolumn{5}{|c|}{$\begin{array}{c}\text { TABLE II } \\
\text { Controller Parameters } \\
\end{array}$} \\
\hline PAR & METER & ADAPTIVE & RISE & ADAPTIVE RISE \\
\hline a1 & & 0.00001 & - & 0.00001 \\
\hline $\mathrm{a} 2$ & & 0.001 & - & 0.001 \\
\hline a3 & & 0.001 & - & 0.001 \\
\hline $\mathrm{a} 4$ & & 0.001 & - & 0.001 \\
\hline$\lambda$ & & 123 & - & 124 \\
\hline $\mathrm{k}$ & & 1 & 0.9 & 0.8 \\
\hline$\alpha_{1}$ & & - & 20 & 2 \\
\hline$\alpha_{2}$ & & - & 1.5 & 1.5 \\
\hline$\beta$ & & - & 3 & 2 \\
\hline
\end{tabular}
varied to check the sensibility of the controllers and are selected by keeping the optimal value between SNR and position error. TABLE III shows the effect of variation of the control parameters on the performance of the controller. 
TABLE III

RMSE (Position and Velocity) and SNR (Position)

\begin{tabular}{lcccccc}
\hline \hline & & ADAPTIVE & & & \multicolumn{2}{c}{ ADAPTIVE RISE } \\
& Position & Velocity & SNR & Position & Velocity & SNR \\
No payload & 0.0528 & 0.2090 & $\mathbf{3 3 . 1 5 6 4}$ & 0.0582 & 0.2200 & $\mathbf{3 7 . 3 2 2 4}$ \\
k reduced by 50 \% & 0.0622 & 0.2132 & 32.1766 & 0.0805 & 0.2451 & 36.5772 \\
k increased by 50\% & 0.0439 & 0.2008 & 32.7794 & 0.0546 & 0.2159 & 36.3614 \\
$\lambda$ reduced by 50 \% & 0.0497 & 0.2110 & 32.7107 & 0.0740 & 0.2394 & 35.6836 \\
$\lambda$ increased by 50\% & $\mathbf{0 . 0 3 4 4}$ & $\mathbf{0 . 1 9 0 7}$ & 32.9310 & $\mathbf{0 . 0 5 4 6}$ & $\mathbf{0 . 2 1 6 5}$ & 36.3743 \\
\hline \hline
\end{tabular}

\section{Real-Time Experimental Results}

To evaluate the performance of the adaptive, RISE and adaptive RISE controllers, comparative analysis is done in different experimental scenarios as shown in Fig. 6, Fig. 7 and Fig. 8. Experiments are done (i) with no payload condition, (ii) with $2.5 \mathrm{Kg}$ payload, (iii) with instant introduction of $0.5 \mathrm{Kg}$ payload between the cycle, (iv) with external disturbances (opposing the joint movement) induced in between cycle, and (v) external disturbances with the instant introduction of $0.5 \mathrm{Kg}$ payload in between cycles.

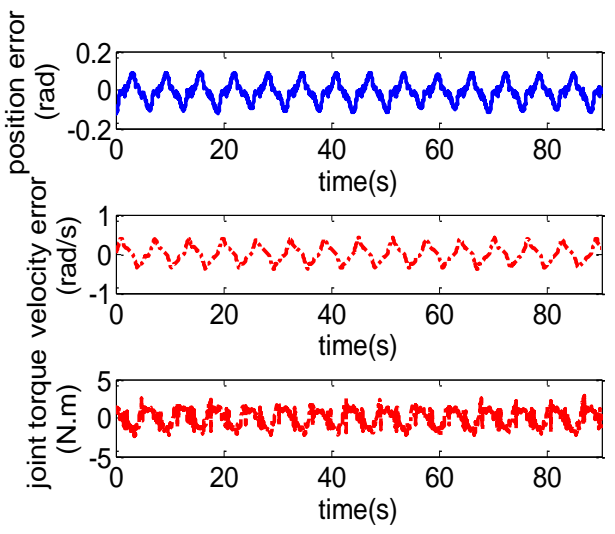

(a) Adaptive

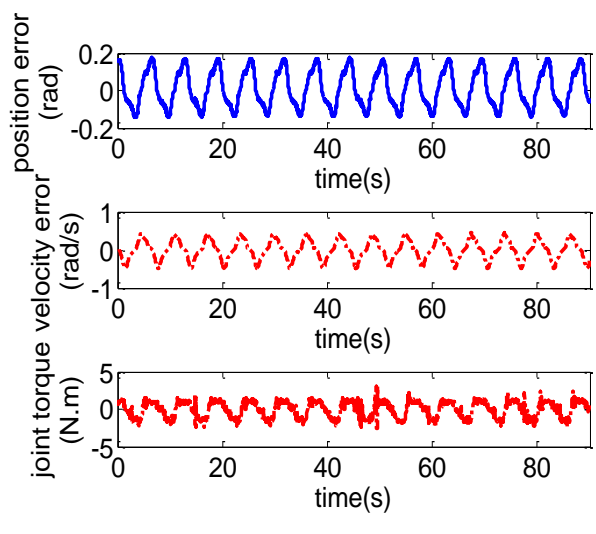

(b) RISE

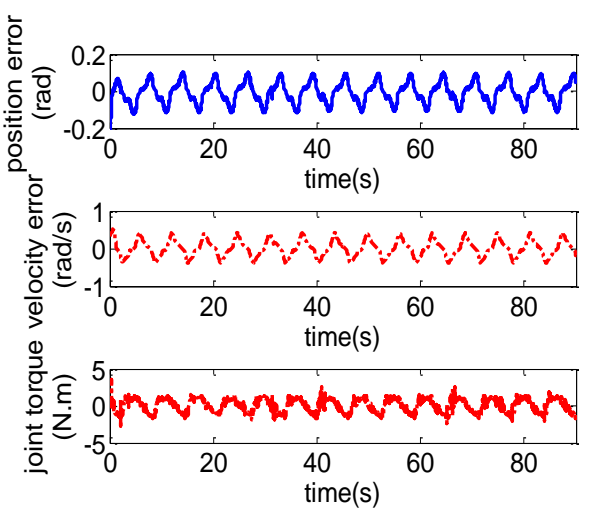

(c) Adaptive RISE

Fig. 6 Torque and Error response for adaptive, RISE and adaptive RISE controllers at no payload condition

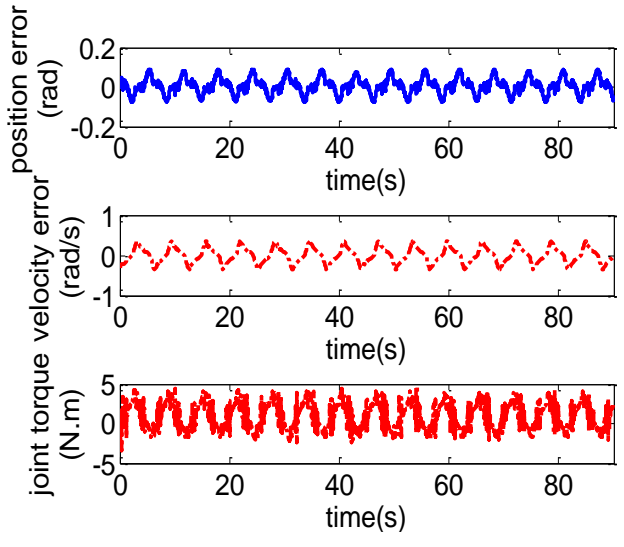

(a) Adaptive

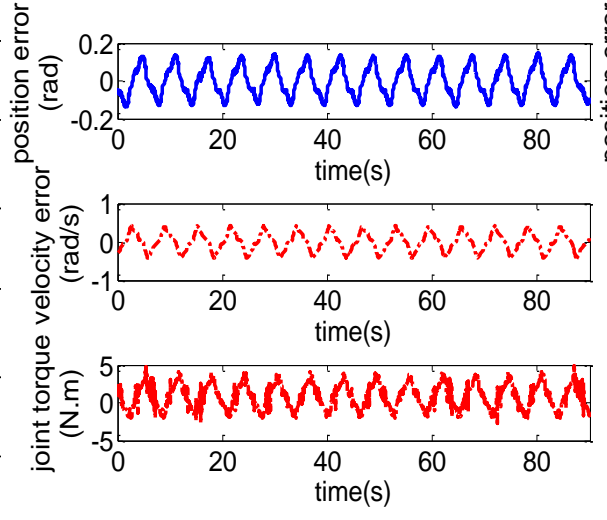

(b) RISE

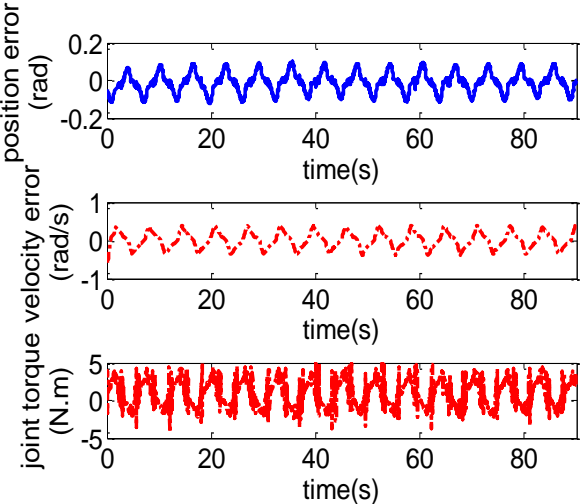

(c) Adaptive RISE

Fig. 7 Torque and Error response for adaptive, RISE and adaptive RISE controllers at constant payload of $2.5 \mathrm{Kg}$ 


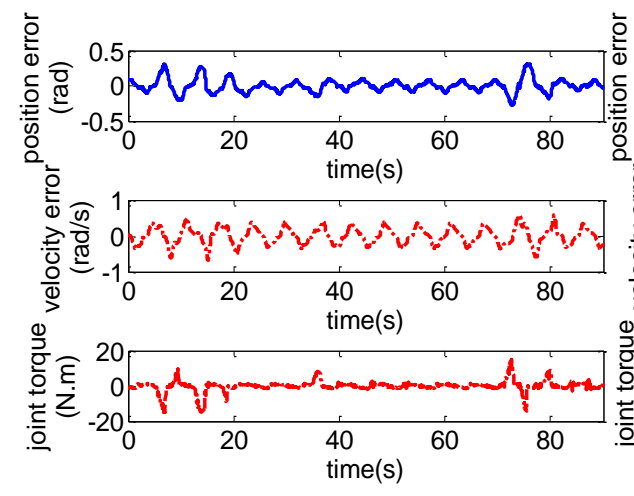

(a) External induced disturbance

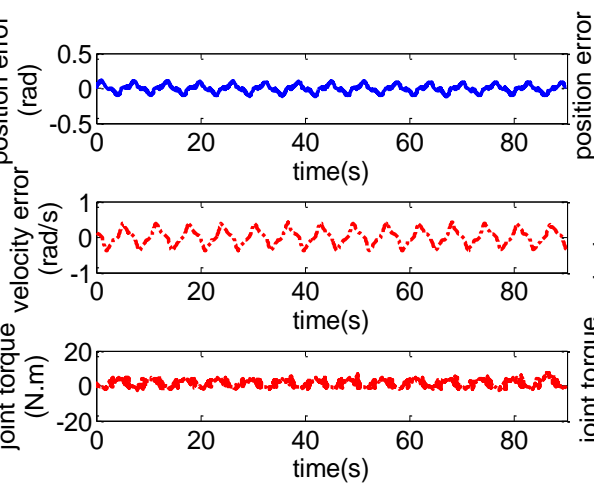

(b) Load Shift

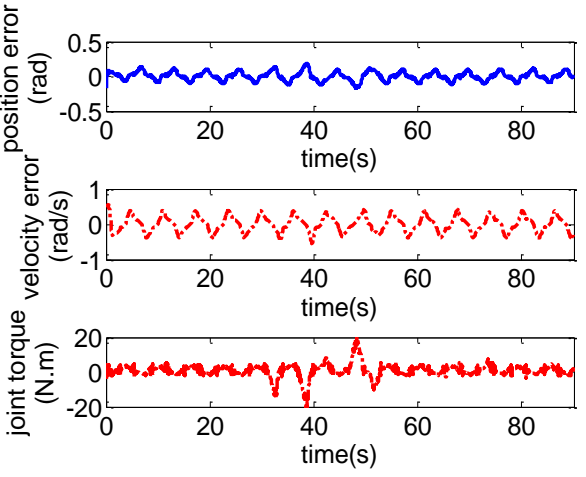

(c) External disturbance with load

Fig. 8 Torque and error response curve for adaptive RISE controller under external induced disturbance, constant load shift and disturbance with load shift

The exoskeleton is fixed on the desk with no contact with the ground. Flexion/Extension movement of the knee joint is considered for the performance analysis. The desired trajectory is a sinusoidal waveform. The root-mean square error (RMSE) in different scenarios are shown in Table III and TABLE IV. The trials are conducted for 90 seconds each with three sets for each scenario. To check the robustness of the controller, tuning parameters are also varied by fifty percent to track the resulting performance. As shown in Fig. 9, all the estimated parameters did not converge to the real values because the aim of the controller here is to maintain the system's stability while maintaining the minimal error and not the parameter identification.

TABLE IV

RMSE (Position and velocity) and SNR (Position) with Load Variations

\begin{tabular}{lcccccccccc}
\multicolumn{1}{c}{ RMSE (Position and velocity) and SNR (Position) with Load Variations } \\
\hline \hline & \multicolumn{3}{c}{ ADAPTIVE } & \multicolumn{4}{c}{ RISE } & \multicolumn{5}{c}{ ADAPTIVE RISE } \\
& Position & Velocity & SNR & Position & Velocity & SNR & Position & Velocity & SNR \\
No payload & 0.0528 & 0.2090 & $\mathbf{3 3 . 1 5 6 4}$ & 0.0987 & 0.2598 & $\mathbf{3 6 . 7 2 0 3}$ & 0.0582 & 0.2200 & $\mathbf{3 7 . 3 2 2 4}$ \\
0.5Kg payload & 0.0552 & 0.2095 & 32.3784 & 0.0891 & 0.2472 & 36.2671 & 0.0568 & 0.2170 & 36.3517 \\
2Kg payload & 0.0333 & 0.2028 & 31.2016 & 0.0694 & 0.2429 & 36.7175 & 0.0461 & 0.2141 & 35.3697 \\
2.5Kg payload & $\mathbf{0 . 0 2 9 8}$ & $\mathbf{0 . 1 9 1 7}$ & 32.1603 & $\mathbf{0 . 0 5 8 6}$ & $\mathbf{0 . 2 3 4 5}$ & 35.6508 & $\mathbf{0 . 0 3 3 1}$ & $\mathbf{0 . 2 0 9 0}$ & 34.0608 \\
\hline \hline
\end{tabular}

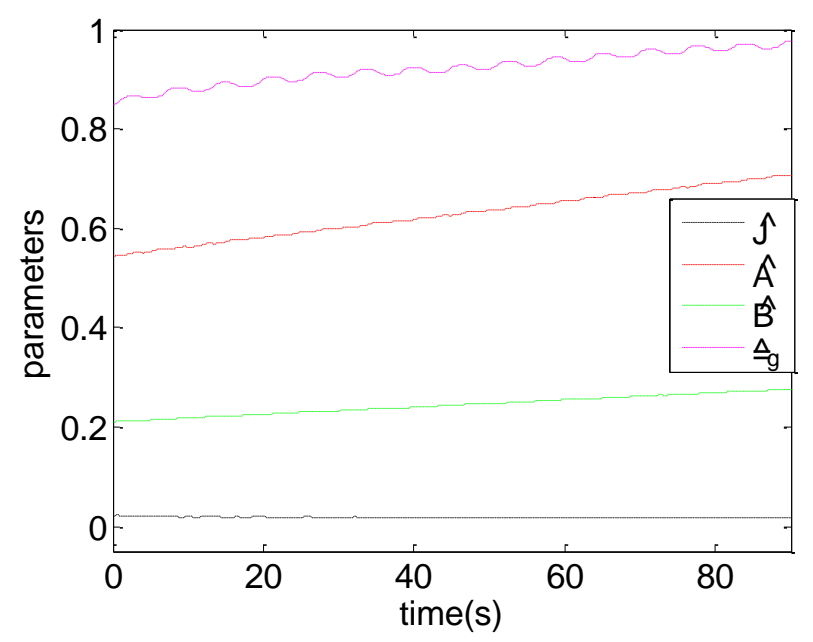

Fig. 9 Adaptive law parameters for Adaptive RISE controller 


\subsection{Scenariol: No payload}

In this scenario, there is no payload on the knee joint and it moves without any external constraint. TABLE IV shows the rootmean square error in position and velocity. The results show that the tracking position error of the adaptive controller is better as compared with the RISE and adaptive RISE controller. But as shown in Fig. 6 there are oscillations in the output (SNR $33.16 \mathrm{~dB}$ ). On the other hand, the RISE controller shows the minimal oscillations (SNR $36.72 \mathrm{~dB}$ ) but there is an increase in tracking error. The adaptive RISE controller shows a slight increase in the tracking error but there is a considerable decrease in the oscillations as compared to the adaptive controller (SNR $37.32 \mathrm{~dB}$ ). Fig. 10 shows the signal to noise ratio (SNR) for adaptive, RISE and adaptive RISE controller. The result shows that the SNR for the adaptive RISE controller comes out to be $37.32 \mathrm{~dB}$ which is higher than of $33.16 \mathrm{~dB}$ for adaptive and $36.72 \mathrm{~dB}$ for RISE controller.

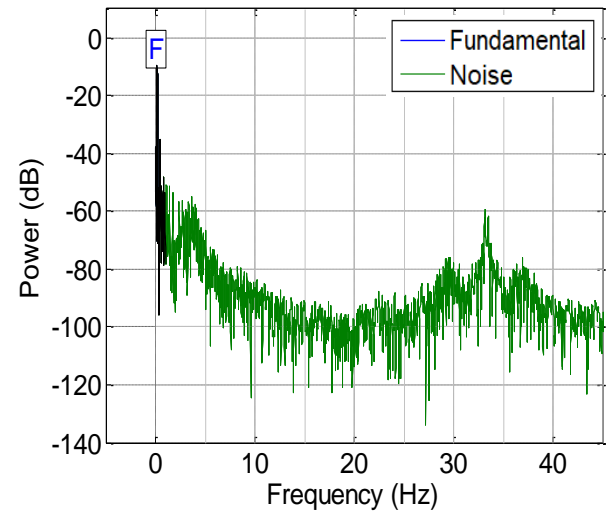

(a) Adaptive

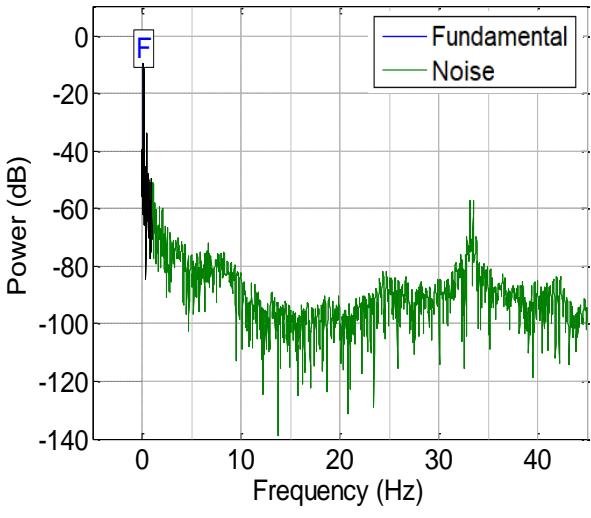

(b) RISE

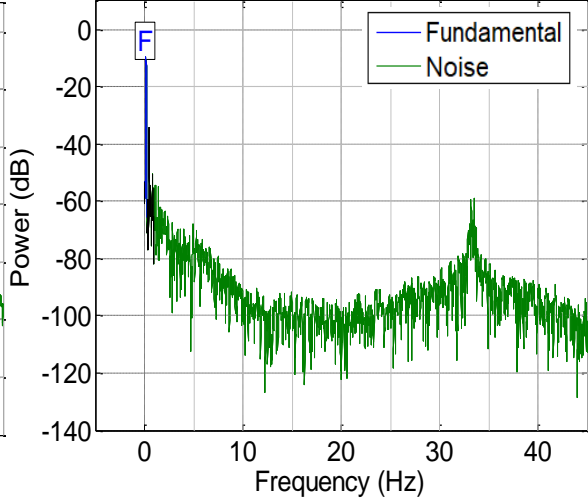

(c) Adaptive RISE

Fig. 10 SNR plots for adaptive, RISE and adaptive RISE controllers

\subsection{Scenario 2: Varying payload}

The payload is introduced on the exoskeleton throughout the flexion/extension cycle. The performance of the controllers is presented in Fig. 7 under loading condition. Similar control performances of the adaptive, RISE and adaptive RISE controllers are found under payload conditions as compared with no payload conditions. TABLE IV summarizes the performance of the controllers. The root mean error for the adaptive RISE controller at $2.5 \mathrm{Kg}$ payload comes out to be 0.0331 which is better than the 0.0586 for the RISE. Similarly, with the other payload conditions, adaptive RISE gives the best solution in terms of compromise between the oscillations and the tracking error.

\subsection{Scenario 3: Instant introduction of load and disturbances}

Three different scenarios are induced to check the performance of the proposed adaptive RISE controller as shown in Fig. 8. Unexpected disturbance may cause instability in the tracking error. The controller must be robust with respect to these external disturbances that may affect the overall stability of the exoskeleton device. In the first condition, external disturbances at different instances (i.e. $5 \mathrm{sec}$ and $75 \mathrm{sec}$ ) are induced during the cycle by opposing the movement of the exoskeleton. As shown, the adaptive 
RISE controller recovers from these disturbances very efficiently. In the second condition, a payload of $0.5 \mathrm{Kg}$ is introduced at 60 seconds during the cycle, where very negligible variation is observed in the error output. In the last condition, the external disturbance and the payload are introduced in the same cycle, the adaptive RISE controller recovers from both disturbances very efficiently.

\section{Conclusion and Future Works}

In this paper, an adaptive RISE controller has been developed to achieve optimal tracking error. The RISE controller is integrated with an adaptive term to compensate for the effects of unstructured disturbances. RISE controller alone uses a high gain value of the feedback. The model-based adaptive term is added to the controller to reduce this control gain, to improve the tracking performance and to introduce the system dynamics in the control structure. A comparative analysis of the tracking response of adaptive, RISE and adaptive RISE controllers is done. The control algorithm of the proposed controller is implemented on EICoSI model of knee exoskeleton. According to the obtained results, the adaptive controller shows the minimal error but there are some oscillations in the output. RISE controller, on the other hand, shows less oscillations but tracking error is more as compared with the adaptive controller. Adaptive RISE controller optimizes the performance between error and oscillations. Quantitively, the tracking error for the adaptive RISE controller is approximately $9 \%$ higher than the adaptive but the SNR is better in adaptive RISE controller by approximately $11 \%$. As compared with RISE controller, the adaptive RISE controller shows better tracking error as well as SNR by approximately 41 and $2 \%$ respectively. Experimental results show that the adaptive RISE controller gives optimal RMS tracking error and signal to noise ratio. To show the robustness of the controller, external disturbances are considered in the control of the exoskeleton. The controller gives the desired performance in terms of tracking error when subjected to these disturbances. The experimental scenarios with payload and disturbances are introduced to mimic the human leg behavior and its possible effects are analyzed. Future work aims at applying the proposed controller on the full lower-body exoskeleton. The desired trajectory movement of the exoskeleton will be calculated using the inverse kinematics. The computation of the human joint torque can also be envisaged using EMG signals of the main lower limb muscles.

\section{References}

[1] A.B. Zoss, H. Kazerooni, A. Chu, Biomechanical design of the Berkeley lower extremity exoskeleton (BLEEX), IEEE/ASME Trans. Mechatronics. 11 (2006) 128-138.

[2] J. Ghan, R. Steger, H. Kazerooni, Control and system identification for the Berkeley lower extremity exoskeleton (BLEEX), Adv. Robot. 20 (2006) 9891014.

[3] M. Vukobratović, B. Borovac, D. Surla, D. Stokić, Biped Locomotion, Springer Berlin Heidelberg, Berlin, Heidelberg, 1990.

[4] H. Kawamoto, Y. Sankai, Comfortable power assist control method for walking aid by HAL-3, in: IEEE Int. Conf. Syst. Man Cybern., IEEE, $2002:$ p. 6.

[5] D.R. Lee, Y.K. Shin, J.H. Park, J.H. You, Concurret Validity and Test-Retest Reliability of the Walkbot-K System for Robotic Gait Training, J. Mech. Med. Biol. 16 (2016).

[6] S.-Y. Kim, L. Yang, I.J. Park, E.J. Kim, M.S. Park, S.H. You, Y.-H. Kim, H.-Y. Ko, Y.-I. Shin, Effects of Innovative WALKBOT Robotic-Assisted Locomotor Training on Balance and Gait Recovery in Hemiparetic Stroke: A Prospective, Randomized, Experimenter Blinded Case Control Study With 
a Four-Week Follow-Up, IEEE Trans. Neural Syst. Rehabil. Eng. 23 (2015) 636-642.

T. Gurriet, S. Finet, G. Boeris, A. Duburcq, A. Hereid, O. Harib, M. Masselin, J. Grizzle, A.D. Ames, Towards Restoring Locomotion for Paraplegics: Realizing Dynamically Stable Walking on Exoskeletons, in: 2018 IEEE Int. Conf. Robot. Autom., IEEE, 2018: pp. $2804-2811$. J.C. Mcleod, S.J. Ward, A.L. Hicks, Evaluation of the Keeogo ${ }^{\mathrm{TM}}$ Dermoskeleton, Disabil. Rehabil. Assist. Technol. (2017) 1-10.

[9] L. Fan, L. Yan, J. Xiao, F. Wang, Dynamics analysis and simulation verification of a novel knee joint exoskeleton, J. Vibroengineering. 19 (2017) 30083018.

[10] K. Suzuki, G. Mito, H. Kawamoto, Y. Hasegawa, Y. Sankai, Intention-based walking support for paraplegia patients with Robot Suit HAL, Adv. Robot. 21 (2007) 1441-1469.

[11] J.E. Pratt, B.T. Krupp, C.J. Morse, S.H. Collins, The RoboKnee: an exoskeleton for enhancing strength and endurance during walking, in: IEEE Int. Conf. Robot. Autom. 2004. Proceedings. ICRA '04. 2004, IEEE, 2004: pp. 2430-2435 Vol.3.

[12] C. Fleischer, H. Gunter, A Human-Exoskeleton Interface Utilizing Electromyography, IEEE Trans. Robot. 5.2a (2008) 872-882.

[13] J. Nikitczuk, B. Weinberg, C. Mavroidis, Control of electro-rheological fluid based resistive torque elements for use in active rehabilitation devices, Smart Mater. Struct. 16 (2007) 418-428.

[14] N. Costa, M. Bezdicek, M. Brown, J.O. Gray, D.G. Caldwell, S. Hutchins, Joint motion control of a powered lower limb orthosis for rehabilitation, Int. J. Autom. Comput. 3 (2006) 271-281.

[15] C. Schmitt, C. Schmitt, P. Métrailler, A. Al-khodairy, R. Brodard, J. Fournier, M. Bouri, R. Clavel, A Study of a Knee Extension Controlled by a Closed Loop Functional Electrical Stimulation, Proc. 9TH Annu. Conf. Int. Funct. Electr. Stimul. Soc. (2004). http://citeseerx.ist.psu.edu/viewdoc/summary?doi=10.1.1.546.7222 (accessed February 4, 2019).

[16] Y. Saito, K. Kikuchi, H. Negoto, T. Oshima, T. Haneyoshi, Development of externally powered lower limb orthosis with bilateral-servo actuator, in: Proc. 2005 IEEE 9th Int. Conf. Rehabil. Robot., IEEE, 2005: pp. 394-399.

[17] Y. Hayashi, K. Kiguchi, Stairs-ascending/descending assist for a lower-limb power-assist robot considering ZMP, in: IEEE Int. Conf. Intell. Robot. Syst., IEEE, 2011: pp. 1755-1760.

[18] K. Kiguchi, T. Tanaka, T. Fukuda, Neuro-fuzzy control of a robotic exoskeleton with EMG signals, IEEE Trans. Fuzzy Syst. 12 (2004) $481-490$.

[19] L.L. Cai, A.J. Fong, C.K. Otoshi, Y. Liang, J.W. Burdick, R.R. Roy, V.R. Edgerton, Implications of Assist-As-Needed Robotic Step Training after a Complete Spinal Cord Injury on Intrinsic Strategies of Motor Learning, J. Neurosci. 26 (2006) 10564-10568.

[20] R. Ekkelenkamp, P. Veltink, S. Stramigioli, H. Van Der Kooij, Evaluation of a virtual model control for the selective support of gait functions using an exoskeleton, in: 2007 IEEE 10th Int. Conf. Rehabil. Robot. ICORR'07, IEEE, 2007: pp. 693-699.

[21] R.J. Sanchez, J. Liu, S. Rao, P. Shah, R. Smith, T. Rahman, S.C. Cramer, J.E. Bobrow, D.J. Reinkensmeyer, Automating arm movement training following severe stroke: Functional exercises with quantitative feedback in a gravity-reduced environment, IEEE Trans. Neural Syst. Rehabil. Eng. 14 (2006) 378-389.

[22] A.H.A. Stienen, E.E.G. Hekman, F.C.T. Van Der Helm, G.B. Prange, M.J.A. Jannink, A.M.M. Aalsma, H. Der Van Kooij, Freebal: Dedicated gravity compensation for the upper extremities, in: 2007 IEEE 10th Int. Conf. Rehabil. Robot. ICORR'07, IEEE, 2007: pp. 804-808.

[23] Meng-Bi Cheng, Ching-Chih Tsai, Hybrid robust tracking control for a mobile manipulator via sliding-mode neural network, in: IEEE Int. Conf. Mechatronics, IEEE, Taipei, Taiwan, 2005: pp. 537-542.

[24] E. Sebastián, M.A. Sotelo, Adaptive fuzzy sliding mode controller for the kinematic variables of an underwater vehicle, J. Intell. Robot. Syst. Theory Appl. 49 (2007) 189-215.

[25] L.I. Lugo-Villeda, A. Frisoli, V. Parra-Vega, M. Bergamasco, Regressor-free force/position control of fixed-base exoskeletons for rehabilitation tasks, in: 2009 IEEE/RSJ Int. Conf. Intell. Robot. Syst. IROS 2009, IEEE, 2009: pp. 1639-1645.

[26] L.I. Lugo-Villeda, A. Frisoli, M. Bergamasco, V. Parra-Vega, Robust Tracking of the Light-Exoskeleton for Arm Rehabilitation Tasks, IFAC Proc. Vol. 42 (2009) 663-668.

[27] S.K. Banala, S.K. Agrawal, Gait Rehabilitation With an Active Leg Orthosis, in: Vol. 7 29th Mech. Robot. Conf. Parts A B, ASME, $2005:$ pp. $459-465$.

[28] B. Weinberg, J. Nikitczuk, S. Patel, B. Patritti, C. Mavroidis, P. Bonato, P. Canavan, Design, control and human testing of an active knee rehabilitation orthotic device, in: Proc. - IEEE Int. Conf. Robot. Autom., IEEE, 2007: pp. 4126-4133.

[29] S. Mohammed, W. Huo, J. Huang, H. Rifaï, Y. Amirat, Nonlinear disturbance observer based sliding mode control of a human-driven knee joint orthosis, Rob. Auton. Syst. 75 (2016) 41-49.

[30] K. Miroslav, K. Petar V, K. Ioannis, Nonlinear and Adaptive Control Design., Wiley, 1995. https://www.wiley.com/enus/Nonlinear+and+Adaptive+Control+Design-p-9780471127321 (accessed November 19, 2018).

[31] S. Sastry, M. Bodson, J.F. Bartram, Adaptive Control: Stability, Convergence, and Robustness, J. Acoust. Soc. Am. 88 (1990) $588-589$. 
[32] L. Peternel, T. Noda, T. Petrič, A. Ude, J. Morimoto, J. Babič, Adaptive control of exoskeleton robots for periodic assistive behaviours based on EMG feedback minimisation, PLoS One. 11 (2016) e0148942.

[33] S. Chen, Z. Chen, B. Yao, X. Zhu, S. Zhu, Q. Wang, Y. Song, Adaptive Robust Cascade Force Control of 1-DOF Hydraulic Exoskeleton for Human Performance Augmentation, IEEE/ASME Trans. Mechatronics. 22 (2017) 589-600.

[34] B. Xian, M.S. Queiroz, D.M. Dawson, A Continuous Control Mechanism for Uncertain Nonlinear Systems, in: IEEE Trans. Automat. Contr., 2004: pp. 251-264.

[35] Z. Cai, M.S. de Queiroz, D.M. Dawson, Robust adaptive asymptotic tracking of nonlinear systems with additive disturbance, IEEE Trans. Automat. Contr. 51 (2006) 524-529.

[36] B. Bidikli, E. Tatlicioglu, A. Bayrak, E. Zergeroglu, A new robust "integral of sign of error" feedback controller with adaptive compensation gain, in: Proc. IEEE Conf. Decis. Control, IEEE, 2013: pp. 3782-3787.

[37] B. Xian, D.M. Dawson, M.S. DeQueiroz, J. Chen, A Continuous Asymptotic Tracking Control Strategy for Uncertain Nonlinear Systems, IEEE Trans. Automat. Contr. 49 (2004) 1206-1211.

[38] N. Fischer, S. Bhasin, W.E. Dixon, Nonlinear control of an autonomous underwater vehicle: A RISE-based approach, in: Proc. 2011 Am. Control Conf., IEEE, San, 2011: pp. 3972-3977.

[39] Z. Wang, A. Behal, Continuous robust control for a class of uncertain MIMO nonlinear systems, in: IEEE Conf. Decis. Control Eur. Control Conf., IEEE, 2011: pp. 7561-7566.

[40] P.M. Patre, W. MacKunis, C. Makkar, W.E. Dixon, Asymptotic Tracking for Systems with Structured and Unstructured Uncertainties, in: Proc. 45th IEEE Conf. Decis. Control, IEEE, 2006: pp. 441-446.

[41] T. Dierks, S. Jagannathan, Neural Network Control of Mobile Robot Formations Using RISE Feedback, IEEE Trans. Syst. Man, Cybern. Part B. 39 (2009) 332-347.

[42] B. Xian, M.S. Queiroz, D.M. Dawson, A Continuous Control Mechanism for Uncertain Nonlinear Systems, in: Optim. Control. Stab. Nonsmooth Anal., Springer, Berlin, Heidelberg, 2004: pp. 251-264.

[43] H. Rifaï, S. Mohammed, B. Daachi, Y. Amirat, Adaptive control of a human-driven knee joint orthosis, in: Proc. - IEEE Int. Conf. Robot. Autom., IEEE, 2012: pp. 2486-2491.

[44] K.J. (Karl J. Åström, R.M. Murray, Feedback systems: an introduction for scientists and engineers, Princeton University Press, 2008. https://press.princeton.edu/titles/8701.html (accessed January 24, 2019).

[45] J. Han, From PID to Active Disturbance Rejection Control, IEEE Trans. Ind. Electron. 56 (2009) 900-906.

[46] S. Mefoued, S. Mohammed, Y. Amirat, Toward Movement Restoration of Knee Joint Using Robust Control of Powered Orthosis, IEEE Trans. Control Syst. Technol. 21 (2013) 2156-2168.

[47] C.-Y. Su, Y. Stepanenko, J. Svoboda, T.P. Leung, Robust adaptive control of a class of nonlinear systems with unknown backlash-like hysteresis, IEEE Trans. Automat. Contr. 45 (2000) 2427-2432.

[48] X.-S. Wang, C.-Y. Su, H. Hong, Robust adaptive control of a class of nonlinear systems with unknown dead-zone, Automatica. 40 (2004) 407-413.

[49] J.A. Blaya, H. Herr, Adaptive Control of a Variable-Impedance Ankle-Foot Orthosis to Assist Drop-Foot Gait, IEEE Trans. Neural Syst. Rehabil. Eng. 12 (2004) 24-31.

[50] J.W. Noble, S.D. Prentice, Adaptation to unilateral change in lower limb mechanical properties during human walking, Exp. Brain Res. 169 (2006) $482-$ 495.

[51] P.-C. Kao, D.P. Ferris, Motor adaptation during dorsiflexion-assisted walking with a powered orthosis, Gait Posture. 29 (2009) $230-236$.

[52] M. Taktak-Meziou, A. Chemori, J. Ghommam, N. Derbel, RISE feedback control for a R/W head track following in hard disc drives, in: 2014 IEEE 11 th Int. Multi-Conference Syst. Signals Devices, SSD 2014, IEEE, 2014: pp. 1-6.

[53] M. Bennehar, A. Chemori, M. Bouri, L.F. Jenni, F. Pierrot, A new RISE-based adaptive control of PKMs: design, stability analysis and experiments, Int. J. Control. 91 (2018) 593-607.

[54] ZATSIORSKY, V., The Mass and Inertia Characteristics of the Main Segments of the Human Body, Biomechanics. (1983) 1152-1159. https://ci.nii.ac.jp/naid/10004066673/ (accessed June 18, 2019).

[55] ZATSIORSKY, V., Estimation of the mass and inertia characteristics of the human body by means of the best predictive regression equations, Biomech. ; Proc. (1985) 233-239. https://ci.nii.ac.jp/naid/10009791260/\#cit (accessed June 18, 2019).

[56] J. Swevers, C. Ganseman, D.B. Tukel, J. de Schutter, H. Van Brussel, Optimal robot excitation and identification, IEEE Trans. Robot. Autom. 13 (1997) 730-740.

[57] N. Fischer, D. Hughes, P. Walters, E.M. Schwartz, W.E. Dixon, Nonlinear RISE-based control of an autonomous underwater vehicle, IEEE Trans. Robot. 


\section{A. Annexure:}

\section{Stability Analysis of the Adaptive RISE Controller}

The stability analysis of the adaptive and RISE controller was presented by Rifai et. al. [43] and Fisher et. al. [57] respectively and for the adaptive RISE controller is given below.

Proposition: Consider the EICoSI exoskeleton model described in equation (2.8) and the proposed controller described in equation (3.18). The controller output ensures that the tracking error remain bounded such that

$$
e_{1} \rightarrow 0 \text { as } \mathrm{t} \rightarrow \infty
$$

Proof: Let $D \subset R^{3 n+p+1}$ is a domain having $y(t) \triangleq\left[z^{T}(t) \tilde{\varphi}^{T}(t) \sqrt{P(t)}\right]=0$ and $\mathrm{P}(\mathrm{t})$ is an auxiliary function expressed by

$$
P(t) \triangleq \beta\left\|e_{2}\left(t_{0}\right)\right\|-e_{2}^{T}(t) N_{d}\left(t_{0}\right)-\int_{t_{0}}^{t} L(\sigma) d \sigma
$$

where, $L(t) \triangleq r^{T}\left(N_{d}(t)-\beta \operatorname{sign}\left(e_{2}\right)\right) \in R$

Assumption 4: The control parameter $\beta$ is chosen such that: $\beta>\xi_{N_{d}}+\left(\frac{\xi_{N_{d}}}{\alpha_{2}}\right)$

$$
\begin{aligned}
& \text { Therefore, } \int_{t_{0}}^{t} L(\sigma) d \sigma \leq \beta\left\|e_{2}\left(t_{0}\right)\right\|-e_{2}^{T}\left(t_{0}\right) N_{d}\left(t_{0}\right) \\
& \Rightarrow P(t) \geq 0, \forall t \geq t_{0}
\end{aligned}
$$

Consider a positive definite continuously differentiable Lyapunov function

$$
V(y, t)=e_{1}^{T} e_{1}+\frac{1}{2} e_{2}^{T} e_{2}+\frac{1}{2} r^{T} M(\theta) r+P+\frac{1}{2} \tilde{\varphi} \Gamma^{-1} \tilde{\varphi}
$$

Assumption 5: The Lyapunov function (V) satisfies the following bounded condition

$$
\begin{aligned}
& \qquad \mathrm{n}_{1}\|y\|^{2} \leq V \leq \mathrm{n}_{2}(\theta)\|y\|^{2} \\
& \text { where, } \mathrm{n}_{1} \triangleq \frac{1}{2} \min \left\{1, \underline{m}, \lambda_{\min }\left\{\Gamma^{-1}\right\}\right\} \\
& \text { and, } \mathrm{n}_{2}(\theta) \triangleq \frac{1}{2} \max \left\{1, \frac{1}{2} \bar{m}(\theta), \frac{1}{2} \lambda_{\max }\left\{\Gamma^{-1}\right\}\right\}
\end{aligned}
$$

$\lambda_{\min }$ and $\lambda_{\max }$ represent the minimum and the maximum eigen values respectively.

Differentiating equation (A.3) yields,

$$
\dot{V}=r^{T} M(\theta) \dot{r}+\frac{1}{2} r^{T} \dot{M}(\theta) r+e_{2}^{T} \dot{e}_{2}+2 e_{1}^{T} \dot{e}_{1}+\dot{P}-\tilde{\varphi}^{T} \Gamma^{-1} \dot{\hat{\varphi}}
$$

Using equations (3.7)-(3.10), (3.22) and (A.1), equation (A.7) can be rewritten as, 


$$
\dot{V}=r^{T} \widetilde{N}(t)-\left(k_{s}+1\right)\|r\|^{2}-\alpha_{2}\left\|e_{2}\right\|^{2}-2 \alpha_{1}\left\|e_{1}\right\|^{2}+2 e_{2}^{T} e_{1}+r^{T} \dot{Y}_{d} \tilde{\varphi}-\tilde{\varphi}^{T} Y_{d} e_{2}
$$

Since, $e_{2}^{T} e_{1} \leq\left(\left\|e_{1}\right\|^{2}+\left\|e_{2}\right\|^{2}\right) / 2, \tilde{\varphi} \leq \varphi+\varphi_{b},\left(\varphi+\varphi_{b}\right)^{T} \xi_{Y_{d}}\left\|e_{2}\right\| \geq 0$ and using equations (3.16) and (3.26), $\dot{V}$ can be upper bounded as

$$
\dot{V} \leq \rho_{1}(\|z\|)\|z\|\left\|e_{2}\right\|-\left(k_{s}+1\right)\|r\|^{2}-\alpha_{2}\left\|e_{2}\right\|^{2}-2 \alpha_{1}\left\|e_{1}\right\|^{2}+\left\|e_{1}\right\|^{2}+\left\|e_{2}\right\|^{2}
$$

where, $\rho_{1}(\|z\|) \triangleq \rho(\|z\|)+\xi_{Y_{d_{2}}}\left(\varphi+\varphi_{b}\right)$

Equation (A.8) can be arranged as

$$
\dot{V} \leq \mathfrak{n}_{3}\|z\|^{2}-\left(k_{s}\|r\|^{2}-\rho_{1}(\|z\|)\|r\|\|z\|\right)
$$

Where $\mathfrak{n}_{3} \triangleq \min \left\{2 \alpha_{1}-1, \alpha_{2}-1,1\right\} . \alpha_{1}$ and $\alpha_{2}$ are chosen accordingly as

$$
\alpha_{1}>\frac{1}{2}, \quad \alpha_{2}>1
$$

On completing the squares of equation (A.10),

$$
\dot{V} \leq \mathfrak{n}_{3}\|z\|^{2}-\frac{\rho_{1}{ }^{2}(z)\left(\|z\|^{2}\right)}{4 k_{s}} \triangleq c\|z\|^{2}
$$

where, $c\|z\|^{2}$ is positive definite continuous function defined on the domain:

$$
D \triangleq\left\{y \in R^{3 n+p+1} \mid\|y\| \leq \rho_{1}^{-1}\left(2 \sqrt{\mathfrak{n}_{3} k_{s}}\right)\right\}
$$

Equations (A.4) and (A.11), are used to prove $V \in \mathcal{L}_{\infty}$ in D. Therefore, $e_{1}, e_{2}, r, \tilde{\varphi} \in \mathcal{L}_{\infty}$ in D. Using equations (3.6)-(3.8), and by applying standard linear analysis method, it can be proved that $\dot{e}_{1}, \dot{e}_{2} \in \mathcal{L}_{\infty}$ and $\theta, \dot{\theta}, \ddot{\theta} \in \mathcal{L}_{\infty}$ in D. Therefore, assumption 2 can be used to show that, $M(\theta), f(\theta, \dot{\theta}), G(\theta), B$ and its derivative $\in \mathcal{L}_{\infty}$ in D. Hence, $\dot{r}(t) \in \mathcal{L}_{\infty}$ in D using equation (A.2). The assumption that $\theta_{d}, \dot{\theta}_{d}, \ddot{\theta}_{d}, \dddot{\theta}_{d}$ exist and are bounded along with assumption 2 can be used to prove $Y_{d}, \dot{Y}_{d}, \ddot{Y}_{d} \in \mathcal{L}_{\infty}$ in D. Equation (3.9) and (3.18) can be used to show that $\tau_{r} \in \mathcal{L}_{\infty}$ and $\dot{\tau}_{r} \in \mathcal{L}_{\infty}$ in D as $r \in \mathcal{L}_{\infty}$. Since $\dot{e}_{1}, \dot{e}_{2}, \dot{r} \in \mathcal{L}_{\infty}$ in D and definitions of $c\left\|_{z}\right\|^{2}$ and $\mathrm{z}(\mathrm{t})$ are used to prove the continuous uniformity of $c\|z\|^{2}$ in D.

Let set $S \subset \mathrm{D}$, and is defined by:

$$
S \triangleq\left\{y \subset D \mid\|z\|^{2} \leq \mathfrak{n}_{1}\left(\rho_{1}^{-1}\left(2 \sqrt{\mathfrak{r}_{3} k_{s}}\right)\right)^{2}\right\}
$$

Using Theorem 8.4 of [58], it can be proved that,

$$
c\|z\|^{2} \rightarrow 0 \text { as } t \rightarrow \infty \forall y\left(t_{0}\right) \in S
$$

(Theorem assumes $\dot{V}(t, x) \leq-W(x) \leq 0$, where $\mathrm{W}(\mathrm{x})$ is positive semidefinite, then all solution of $\dot{x}=f(t, x)$ with invariant set $\left\{x \in R^{n} \mid W(x)=0\right\}$ are bounded and satisfy $W(x(t)) \rightarrow 0$ as $\left.t \rightarrow \infty\right)$

Definition of $\mathrm{z}(\mathrm{t})$ and equation (A.13) can be used to conclude that

$$
\|e(t)\| \rightarrow 0 \text { as } t \rightarrow \infty \forall y\left(t_{0}\right) \in S
$$


The equation above proves that the system is asymptotically stable. 